\title{
Modulation of Kekulé adatom ordering due to strain in graphene
}

DOI:

10.1103/PhysRevB.97.165430

\section{Document Version}

Final published version

Link to publication record in Manchester Research Explorer

\section{Citation for published version (APA):}

González-Árraga, L., Guinea, F., \& San-Jose, P. (2018). Modulation of Kekulé adatom ordering due to strain in graphene. Physical Review B, 97(16), [165430]. https://doi.org/10.1103/PhysRevB.97.165430

\section{Published in:}

Physical Review B

\section{Citing this paper}

Please note that where the full-text provided on Manchester Research Explorer is the Author Accepted Manuscript or Proof version this may differ from the final Published version. If citing, it is advised that you check and use the publisher's definitive version.

\section{General rights}

Copyright and moral rights for the publications made accessible in the Research Explorer are retained by the authors and/or other copyright owners and it is a condition of accessing publications that users recognise and abide by the legal requirements associated with these rights.

\section{Takedown policy}

If you believe that this document breaches copyright please refer to the University of Manchester's Takedown Procedures [http://man.ac.uk/04Y6Bo] or contact uml.scholarlycommunications@manchester.ac.uk providing relevant details, so we can investigate your claim.

\section{OPEN ACCESS}




\title{
Modulation of Kekulé adatom ordering due to strain in graphene
}

\author{
L. González-Árraga, ${ }^{1}$ F. Guinea, ${ }^{1,2}$ and P. San-Jose ${ }^{3}$ \\ ${ }^{1}$ IMDEA Nanociencia, Calle de Faraday 9, 28049 Madrid, Spain \\ ${ }^{2}$ Department of Physics and Astronomy, University of Manchester, Manchester M13 9PL, United Kingdom \\ ${ }^{3}$ Materials Science Factory, ICMM-CSIC, Sor Juana Ines de La Cruz 3, 28049 Madrid, Spain
}

(Received 20 December 2017; revised manuscript received 16 February 2018; published 23 April 2018)

\begin{abstract}
Intervalley scattering of carriers in graphene at "top" adatoms may give rise to a hidden Kekulé ordering pattern in the adatom positions. This ordering is the result of a rapid modulation in the electron-mediated interaction between adatoms at the wave vector $\boldsymbol{K}-\boldsymbol{K}^{\prime}$, which has been shown experimentally and theoretically to dominate their spatial distribution. Here we show that the adatom interaction is extremely sensitive to strain in the supporting graphene, which leads to a characteristic spatial modulation of the Kekulé order as a function of adatom distance. Our results suggest that the spatial distributions of adatoms could provide a way to measure the type and magnitude of strain in graphene and the associated pseudogauge field with high accuracy.
\end{abstract}

DOI: 10.1103/PhysRevB.97.165430

\section{INTRODUCTION}

Much of the rich physics of graphene stems from the peculiarities of its intrinsic electronic structure, such as its gapless Dirac spectrum, the chirality of its carriers, and the emergence of pseudogauge fields as a result of inhomogeneous strains [1-3]. These are all 'intravalley-' properties, defined independently within valleys $\boldsymbol{K}$ and $\boldsymbol{K}^{\prime}$. They are responsible for, e.g., graphene's high mobilities [4], Klein tunneling [5], the valley-Hall effect [6], and the emergence of topologically protected boundary states in bilayers $[7,8]$. They remain robust as long as valley symmetry is preserved, i.e., as long as any perturbation or disorder present in the sample acts symmetrically on the two sublattices of the crystal. Atomiclike defects are one important type of perturbation that does not, in general, preserve valley symmetry and allows for scattering events with an intervalley $\Delta \boldsymbol{K}=\boldsymbol{K}-\boldsymbol{K}^{\prime}$ momentum transfer ( $\hbar=1$ ) [9].

Intervalley scattering may be important at the edges of a generic graphene flake [10,11], at substitutional dopants [12-14], and at certain adatoms [15] that adsorb to graphene in a "top" configuration (i.e., adsorbed atop individual carbon atoms), such as fluor [16] and hydrogen [17], thereby breaking sublattice symmetry. Despite destroying the chiral nature of carriers in graphene, intervalley scattering is also fundamentally interesting in its own right [18] and can actually become a powerful tool, particularly for graphene functionalization. It is crucial for the engineering of enhanced spin-orbit couplings [15] and finite band gaps in graphene via decoration with adatoms [19-22] by the effect of a crystalline substrate [23-25] or through electron-phonon interaction [26].

Here we focus on another striking effect of intervalley scattering, the unique ordering mechanism of top adatoms [20] and similar atomiclike defects $[27,28]$ in graphene. Ordering results from the electron-mediated interactions between defects as graphene quasiparticles scatter between them [29-31]. Scattering at adatoms locally modifies the electronic density of states in graphene, which gives rise to Friedel oscillations $[12,32,33]$ and to a change in the total electronic energy that depends on the distance between adatoms. Analogous mechanisms exist also in more conventional metallic surfaces [34,35]. In graphene, this gives rise to a fermionic analog of the Casimir force [36] and has been shown to be the dominant contribution in the interaction between graphene adatoms [37]. It leads to the self-organization of atomic defects and adatoms at different levels, including sublattice ordering [21,31], Kekulé ordering [30], and spatial clustering [29]. Kekulé ordering, recently demonstrated in experiment [28], is probably the most striking of these. In this work we show that electron-mediated Kekulé ordering is extremely sensitive to elastic strains in the underlying graphene. The connection arises from the effect of strain-induced pseudogauge fields on intervalley scattering and could provide a sensitive way to measure strains through adatom distributions or, conversely, to control Kekulé ordering of adatoms through strain engineering.

\section{FORMALISM}

Consider a top adatom on sublattice $\sigma=\mathrm{A}$, B of a graphene unit cell centered at $\boldsymbol{r}=n_{1} \boldsymbol{a}_{1}+n_{2} \boldsymbol{a}_{2} \quad\left(\boldsymbol{a}_{i}\right.$ are graphene's lattice vectors with $\left|\boldsymbol{a}_{i}\right|=a_{0}$ and $\left.|\Delta \boldsymbol{K}|=8 \pi / \sqrt{3} a_{0}\right)$. One may classify such an adatom by the sublattice $\sigma$ and an integer Kekulé index $v_{\boldsymbol{r}}$, such that $\Delta \boldsymbol{K} \cdot \boldsymbol{r}=2 \pi v_{\boldsymbol{r}} / 3+2 \pi n$ for some integer $n$, i.e.,

$$
v_{\boldsymbol{r}}=\frac{\Delta \boldsymbol{K} \cdot \boldsymbol{r}}{2 \pi / 3} \bmod 3=\left(n_{1}-n_{2}\right) \bmod 3=0,1,2 .
$$

These three possibilities are color-coded as red, green, and blue here and are shown in Fig. 1 for one of the graphene sublattices. Hidden Kekulé order [28] consists of collections of top adatoms or atomic defects which minimize their quasiparticlemediated interaction energy by adopting the same values of $v_{r}$ and (possibly) the same value of $\sigma$ (see yellow adatoms in Fig. 1). We now describe the mechanism that gives rise to Kekule ordering and then analyze how it is affected by the presence of elastic strains.

The interaction between two adatoms on graphene has various contributions, including local elastic deformations of graphene around adatoms, direct overlap of adatom orbitals, 

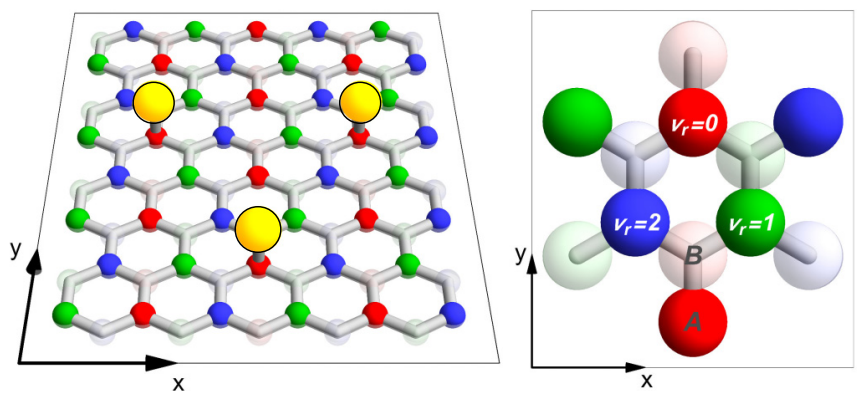

FIG. 1. Sketch of the Kekulé index $v_{r}=0,1,2$ (red, green, blue) of sites on each (A/B) sublattice in graphene. Hidden Kekulé ordering of top adatoms (in yellow) corresponds to adsorption on sites with equal $v_{r}$ (here $v_{r}=0$ ) as a result of adatom interaction mediated by carriers in graphene that undergo intervalley scattering. Sublattice correlation of adatoms may also arise from the same interaction.

direct Coulomb interactions (monopolar or multipolar), and interactions mediated by scattering of quasiparticles in graphene. Of these, only the last two are relevant in realistic conditions [37], with the latter dominating the interaction of neutral adatoms. Direct Coulomb interactions are rather simple and do not produce any Kekulé ordering, so we will concentrate on the far richer properties of the electron-mediated interaction potential $U(\boldsymbol{r})$. We model the graphene-adatom system in a tight-binding approximation,

$$
H=-t \sum_{\langle i, j\rangle} c_{i}^{\dagger} c_{j}+\epsilon_{0} \sum_{k} d_{k}^{\dagger} d_{k}-t^{\prime} \sum_{k}\left(d_{k}^{\dagger} c_{k}+c_{k}^{\dagger} d_{k}\right),
$$

where $c_{i}$ are graphene $\pi$ orbitals and $d_{k}$ are adatom states, located at positions $\boldsymbol{r}_{k}$ and coupled to a single $c_{k}$ state in graphene (top configuration). Consider for simplicity only two adatoms in the system $k=1,2$ on sublattices $\sigma_{1}$ and $\sigma_{2}$ at a distance $\boldsymbol{r}=\boldsymbol{r}_{2}-\boldsymbol{r}_{1}$. The interaction potential $U_{\sigma_{1} \sigma_{2}}(\boldsymbol{r})$ can be written $[29,31,38]$ as the total energy of all the electrons in the system, as they adjust to the presence of the adatoms,

$$
\begin{aligned}
U_{\sigma_{1} \sigma_{2}}(\boldsymbol{r}) & =\int d \omega\left[\rho(\omega)-\rho_{\infty}(\omega)\right] \omega f(\omega), \\
\rho(\omega) & =-\frac{1}{\pi} \operatorname{Tr}\left[G_{1}^{\sigma_{1}}(\omega)+G_{2}^{\sigma_{2}}(\omega)+\int d^{2} r^{\prime} G\left(\boldsymbol{r}^{\prime}, \boldsymbol{r}^{\prime} ; \omega\right)\right] .
\end{aligned}
$$

Here $G$ and $G_{k}^{\sigma_{k}}$ are the full, retarded Green's functions of graphene and the two adatoms, respectively, and $f(\omega)$ is the Fermi function (for concreteness, zero temperature and zero filling are assumed from now on). The potential depends implicitly on the adatom distance $\boldsymbol{r}$ and contains fast spatial harmonics $\sim \cos (\Delta \boldsymbol{K} \cdot \boldsymbol{r})$ due to the interference of $\boldsymbol{K}$ and $\boldsymbol{K}^{\prime}$ that results from intervalley scattering. The reference density of states $\rho_{\infty}(\omega)$ is defined as the limit for $r \rightarrow \infty$, so that $U(\boldsymbol{r} \rightarrow \infty)=0$.

\section{RESULTS AND DISCUSSION}

We computed $U(\boldsymbol{r})$ numerically to all orders in the coupling $t^{\prime}$, as described in Appendix A. Calculations were performed using version 0.6 of the Julia language [39]. In the weakcoupling limit the results agree with analytical expressions for $U$ that were obtained in the literature for unstrained graphene
[31]. It was shown, using a simplified adatom model, that in the limit of weakly coupled adatoms, $U$ exhibit a Kekulé modulation given by

$$
\begin{gathered}
U_{A A}(\boldsymbol{r})=U_{B B}(\boldsymbol{r}) \approx v_{A A}(|\boldsymbol{r}|) \cos ^{2}\left(\pi v_{\boldsymbol{r}} / 3\right), \\
U_{A B}(\boldsymbol{r})=U_{B A}(\boldsymbol{r}) \approx v_{A B}(|\boldsymbol{r}|) \sin ^{2}\left(\pi v_{\boldsymbol{r}} / 3+\phi_{\boldsymbol{r}}\right) .
\end{gathered}
$$

Here $\phi_{\boldsymbol{r}}$ is the angle between $\Delta \boldsymbol{K}$ and $\boldsymbol{r}=\boldsymbol{r}_{2}-\boldsymbol{r}_{1} . v_{A A}(r)$ and $v_{A B}(r)$ are smooth functions of interadatom distance that in the absence of dissipation fall as $1 / r^{3}$ at long distances. The sign of $v_{\sigma \sigma^{\prime}}(r)$ is controlled by the adatom coupling $t^{\prime}$, and the interaction strength and decay with $r$ are strongly affected by inelastic processes in graphene (see Appendixes D and $\mathrm{B}$ for further discussion). This results in a rich and partially tunable interaction phenomenology between adatoms. Perhaps the most relevant property, however, is that for weak couplings, $v_{A A}(r)$ is attractive and $v_{A B}(r)$ is repulsive, while for strong coupling the opposite is true. Hence, adatoms will exhibit ferro- or antiferrolike order in the sublattice quantum number, depending on how strongly coupled they are to graphene. The Kekule factor, in contrast, is much more universal, and the expressions in Eqs. (4) and (5) remain qualitatively correct regardless of coupling strength. Corrections come in the form of weaker harmonics in the angular coordinate $\phi_{\boldsymbol{r}}$. The Kekule factor effectively produces six different, spatially smooth potential components $U_{\sigma_{1}, \sigma_{2}, v_{r_{1}}, v_{r_{2}}}$ for each combination of $\sigma, \sigma^{\prime}$ and $v_{\boldsymbol{r}_{1}}, \nu_{\boldsymbol{r}_{2}}$. In the following, we will focus on these components to reveal the favored Kekule and sublattice ordering in each case.

Numerical results for $U_{A A}(\boldsymbol{r})$ and $U_{A B}(\boldsymbol{r})$ without strain are shown in Figs. 2(a) and 2(b), respectively. Parameters are chosen in the weak-coupling regime, which corresponds to the phenomenology seen in the experiment of Ref. [28]. In the insets we show the three Kekulé components corresponding to the three values of $v_{\boldsymbol{r}}=v_{\boldsymbol{r}_{1}-\boldsymbol{r}_{2}}=0,1,2$ (red, green, and blue). The main panels show all the Kekulé components together in real space but are plotted to emphasize the color of the most (least) favored Kekulé component at each adatom distance for $U_{A A}\left(U_{A B}\right)$. Points with a more negative potential $U_{A A}(\boldsymbol{r})$ are rendered last in Fig. 2(a), so that the most visible color of a given point corresponds to the Kekule character of the potential minimum. For $U_{A B}(\boldsymbol{r})$ in Fig. 2(b) we use the opposite rendering order, so that the Kekule character of points with the strongest repulsion is the most visible. Cuts of the potential along the vertical and horizontal directions are also included. We note that $U_{A A}$ at $v_{\boldsymbol{r}}=0$ (red) is the most attractive potential component [topright inset in Fig. 3(a)]. In the chosen parameter regime the electron-mediated potential favors isotropic configuration of adatoms on the same sublattice $\sigma$ and with equal Kekule index. Note that the angular profile of all potential components follows Eqs. (4) and (5).

We now consider the same problem in the presence of uniform strain $\epsilon_{i j}$ in graphene, such that the position of each carbon atom $\boldsymbol{r}_{i}$ becomes $\boldsymbol{r}_{i}+\boldsymbol{\epsilon} \cdot \boldsymbol{r}_{i}$. The distortion is incorporated into the tight-binding description of Eq. (2) by making the hopping $t$ depend on the carbon-carbon distance as $t_{\epsilon}=$ $t \exp \left[-\beta\left(\left|\boldsymbol{r}_{i}-\boldsymbol{r}_{j}\right| / a-1\right)\right]$, where $\beta \approx 3$ and $a=a_{0} / \sqrt{3}$ is the unstrained carbon-carbon distance. For realistic strains, this shifts the $\boldsymbol{K}$ and $\boldsymbol{K}^{\prime}$ valleys by an opposite pseudogauge vector 
(a)

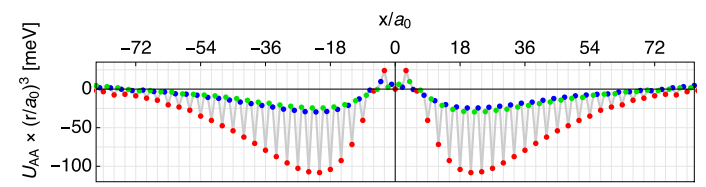

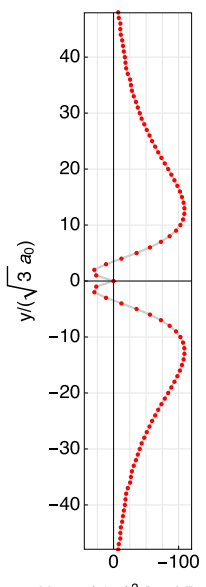

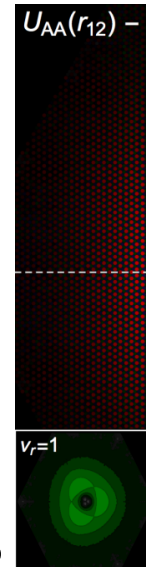

$U_{\mathrm{AA}} \times\left(\mathrm{r} / a_{0}\right)^{3}[\mathrm{meV}]$

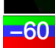

$-60$

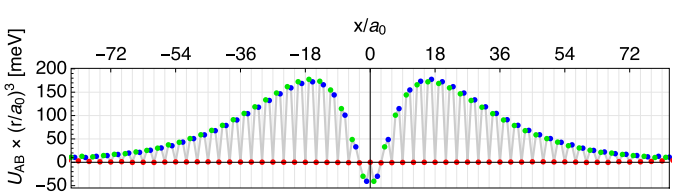

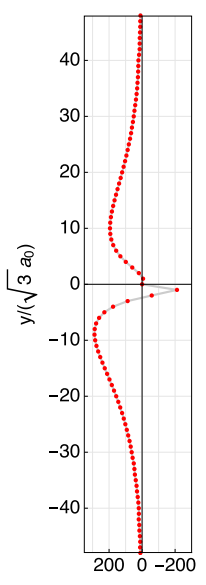

$U_{\mathrm{AB}} \times\left(\mathrm{r} / \mathrm{a}_{0}\right)^{3}[\mathrm{meV}]$

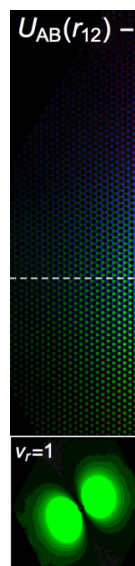

$-60$
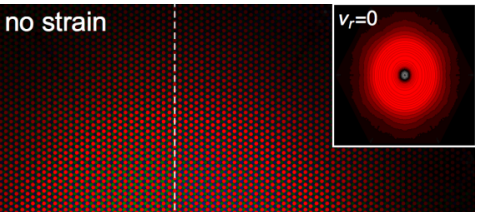

(b)

FIG. 2. Interaction potential between two adatoms (with $\epsilon_{0}=$ $-0.15 t)$ on unstrained graphene, weakly attached $\left(t^{\prime}=0.7 t\right)$ to (a) the same and (b) different subblattices. The interaction potential results from the scattering of graphene carriers at the adatom sites. The potential is attractive for equal sublattices and repulsive otherwise. By color-coding the potential according to the Kekulé character $v_{r}$ of the separation vector $\boldsymbol{r}=\boldsymbol{r}_{2}-\boldsymbol{r}_{1}$, we see that at equilibrium the two adatoms rest on the same sublattice at sites with equal Kekule character $\left[v_{r}=0(\mathrm{red}) \Rightarrow v_{r_{1}}=v_{r_{2}}\right]$. Regardless of the radial dependence of the interaction potential [multiplied here by $\left(|\boldsymbol{r}| / a_{0}\right)^{3}$ for visibility], its Kekulé components (colored insets) satisfy the simple forms in Eqs. (4) and (5), up to small corrections from higher angular harmonics.

\section{$[1,3,40]$}

$$
\boldsymbol{A}= \pm \frac{2 \beta}{\sqrt{3} a_{0}}\left(\epsilon_{x x}-\epsilon_{y y},-2 \epsilon_{x y}\right)
$$

(the $\hat{x}$ axis corresponds here to the zigzag direction). In the case of homogeneous strain, this pseudogauge potential is of no consequence to intravalley physics, as it can be gauged away. It has, however, a strong impact in intervalley scattering since the Kekulé momentum transfer changes to $\Delta \boldsymbol{K}+2 \boldsymbol{A}$. Consequently, it would be natural to expect intervalleydependent quantities such as $U_{\sigma \sigma^{\prime}}(r)$ to exhibit signatures of a uniform strain. The weak-coupling Kekulé should then become

$$
\begin{aligned}
& U_{A A}(\boldsymbol{r}) \approx v_{A A}(|\boldsymbol{r}|) \cos ^{2}\left(\pi v_{\boldsymbol{r}} / 3+\boldsymbol{A} \cdot \boldsymbol{r}\right), \\
& U_{A B}(\boldsymbol{r}) \approx v_{A B}(|\boldsymbol{r}|) \sin ^{2}\left(\pi v_{\boldsymbol{r}} / 3+\boldsymbol{A} \cdot \boldsymbol{r}+\phi_{\boldsymbol{r}}\right) .
\end{aligned}
$$

This expectation is indeed confirmed by our numerical simulations. Figure 3 shows the modified potentials $U_{A A}$ [Figs. 3(a)-3(c)] and $U_{A B}$ [Figs. 3(d)-3(f)] for the same parameters as in Fig. 2 under a uniform 1\% uniaxial strain along the $x$ and $y$ directions and a $1 \%$ uniform shear strain. We concentrate on the $U_{A A}(\boldsymbol{r})$ potential, as $U_{A B}$ remains repulsive and is thus irrelevant for the equilibrium adatom configurations (see Appendix B for additional results in the case of strong coupling). The equal-sublattice configuration is still the most stable one in the presence of strain in this regime. One immediately observes, however, a new spatial modulation in each of the Kekulé components that is linear in $\epsilon_{i j}$. While a uniform Kekulé adatom configuration $v_{r}=0$ was favored in the case without strains, a $1 \%$ strain makes the potential minimum change Kekule character with distance, precessing between $v_{r}=0,1,2$ (red, green, blue) as the two adatoms are separated [see vertical and horizontal stripes in Figs. 3(a)-3(c)]. This type of precessing interaction is reminiscent of the Dzyaloshinskii-Moriya exchange interactions in chiral magnets [41,42], responsible for the formation of skyrmion spin structures [43], although here it operates in the Kekulé instead of the spin sector.

The spatial modulation is consistent with the form of $\boldsymbol{A}$ given in Eq. (6). Uniaxial strains $\epsilon_{x x}$ and $\epsilon_{y y}$ along the $x$ and $y$ directions both modulate the Kekulé character along the $x$ direction, albeit in an opposite sequence order. In contrast, a shear strain $\epsilon_{x y}$ creates a modulation along the $y$ direction, with a period that is half that of the uniaxial strain. The modulation period is given by $\pi /|6 A|$, i.e., around $3-4 \mathrm{~nm}$ for $1 \%$ uniaxial strain.

At large distances, particularly if adatom binding is weak, two isolated adatoms may interact very weakly due to the $\sim\left(r / a_{0}\right)^{3}$ scaling of the potential. Hence, our predictions in this case may be relevant only at rather low temperatures. For a large ensemble of adatoms, however, the relevant adatom distance is given by the typical adatom separation, given by their surface density. Unless the density is very low, the Kekulé orientation of domains should become robust at much larger temperatures in this case since the interactions with all adatoms within a given range add up constructively. Kekulé order in clusters is, moreover, expected to also exhibit the kind of spatial modulation described here with strain. The long-range coherence of Kekulé domains will be controlled by the long-range component of the interaction, so striped Kekulé domains are expected to form even under weak uniform strains. This effect could be tested under the conditions of the experiment of Gutierrez et al. [28] (Kekulé domain sizes in the tens of nanometers and above, substantially greater than 
(a)
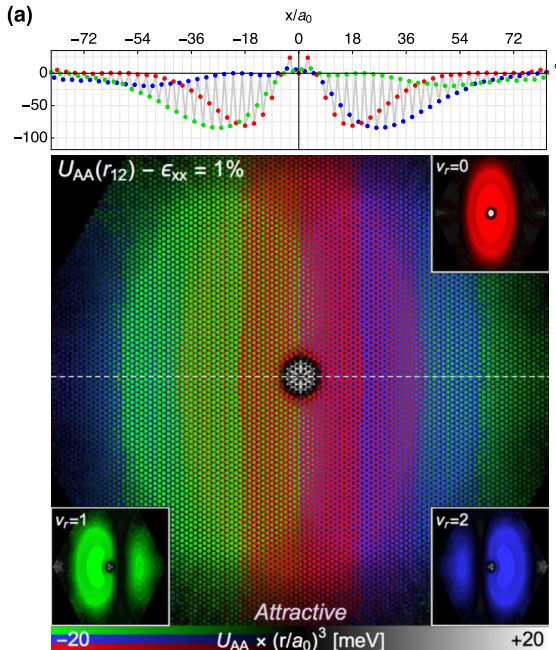

(d)
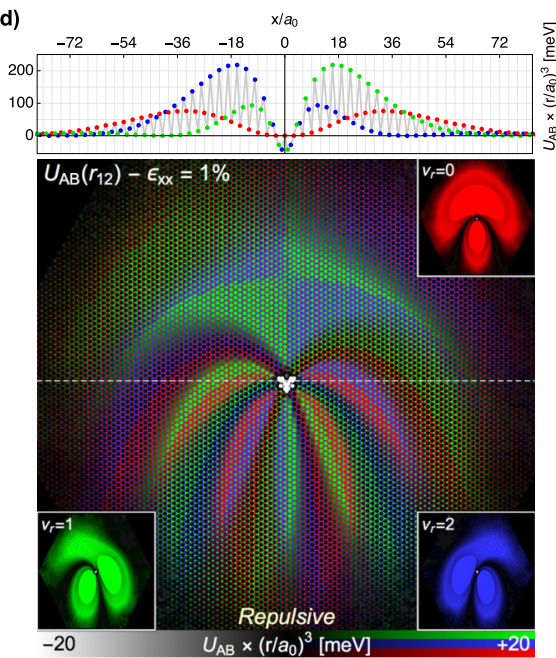

(b)
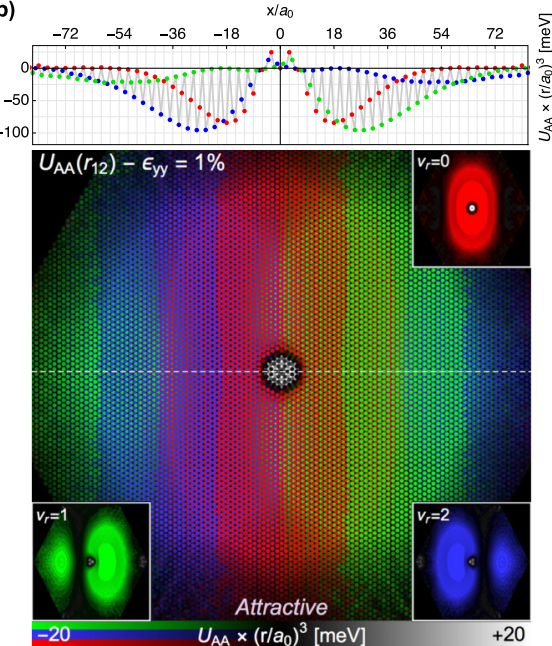

(e)
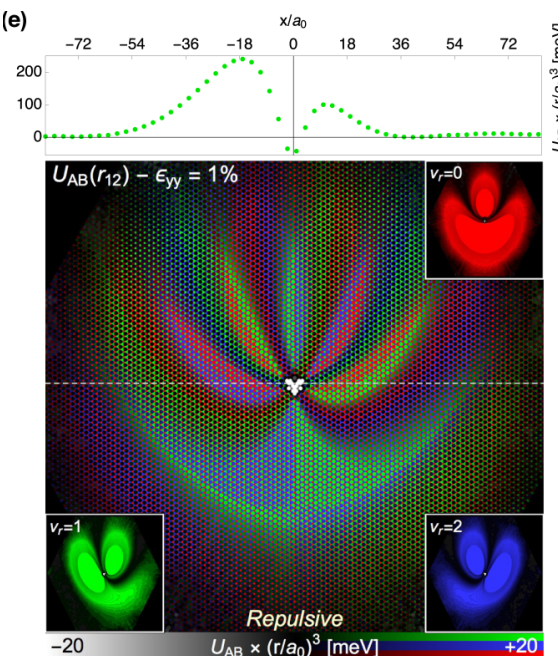
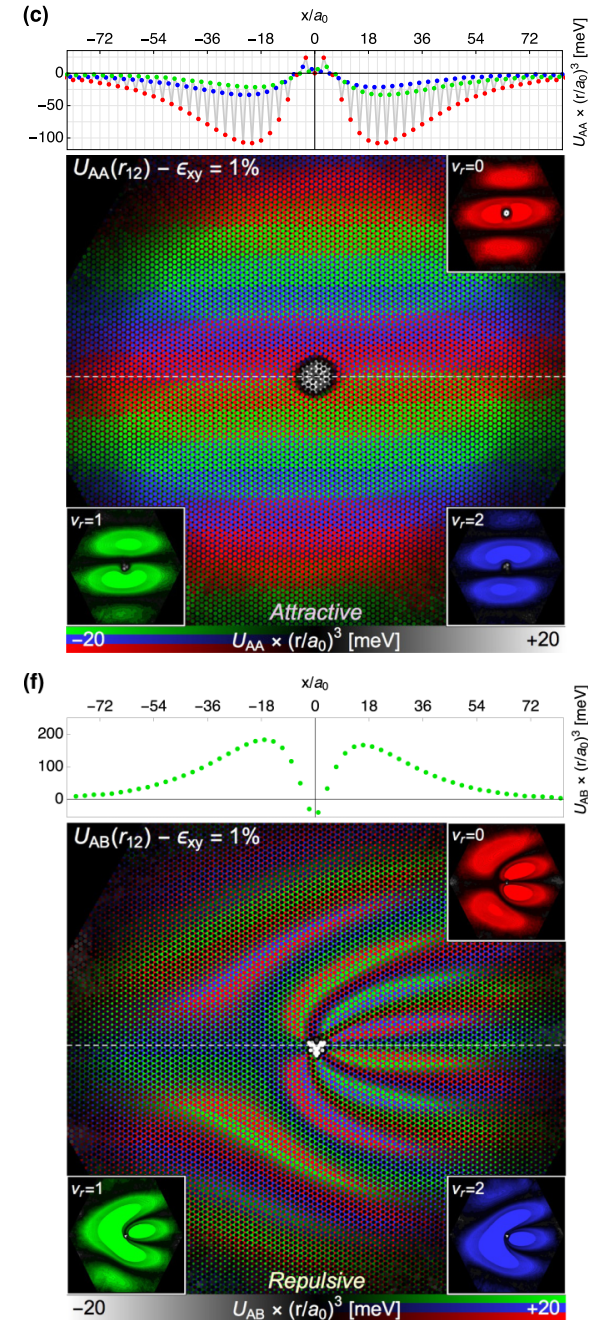

FIG. 3. Interaction potential between two adatoms as in Fig. 2, with a $1 \%$ uniform strain present in graphene, either (a) and (d) uniaxial $\epsilon_{x x}$, (b) and (e) uniaxial $\epsilon_{y y}$, or (c) and (f) a uniform shear $\epsilon_{x y}$. Equal-sublattice configurations are still preferred, but the Kekulé character acquires a modulation with distance that reflects the change in the intervalley separation $\boldsymbol{K}-\boldsymbol{K}^{\prime}$ by a pseudogauge field $2 \boldsymbol{A} \sim\left(\epsilon_{x x}-\epsilon_{y y},-2 \epsilon_{x y}\right)$.

modulation periods at $1 \%$ strains). In such cases the spatial modulation of Kekulé alignment is expected to show a high sensitivity to the magnitude and type (uniaxial/shear) of strains in the sample.

\section{CONCLUSION}

We have concentrated here on the simplest case of a pointlike adatom in a top configuration. More complex adsorbates, such as larger molecules or adatoms in different stacking configurations (hollow and bridge), should be expected to result in different interaction potentials. Likewise, the inclusion of additional physical ingredients, such as electronic interactions and adatom magnetism, could extend the results presented here. We explore a number of these extensions in the Appendixes B and C (strong coupling, on-site interactions, adatom magnetization, and RKKY exchange [19,4446]). While quantitative differences were found, they were mostly confined to the range and sign of the different smooth Kekule components $v_{\sigma \sigma^{\prime}}(r)$. The Kekulé modulation of the potential and its dependence on strain, Eqs. (7) and (8), remain essentially unchanged. The fundamental connection between
Kekule order and strain is thus found to be universal and is one of the most striking manifestations of uniform strains in graphene.

\section{ACKNOWLEDGMENTS}

L.G.-A. and F.G. acknowledge the financial support from the Marie-Curie-Initial Training Networks (ITN) program through Grant No. 607904-SPINOGRAPH. P.S.-J. acknowledges financial support from the Spanish Ministry of Economy and Competitiveness through Grant No. FIS2015-65706-P (MINECO/FEDER).

\section{APPENDIX A: INTERACTION POTENTIAL BETWEEN TWO ADATOMS}

The total interaction energy between two identical adatoms in a top configuration on a graphene monolayer can be decomposed in several contributions. Solenov et al. showed [37] that the dominant contributions are reduced to two: the electrostatic repulsion and the interaction mediated by electron scattering in graphene. The first may be present even for charge-neutral 
adatoms in multipolar form but is otherwise rather simple. The second results in a much richer structure of the interaction and has been shown to strongly dominate the ordering of impurities in some situations.

The main feature of the electron-mediated interaction $U(\boldsymbol{r})$ between adatoms on graphene is that, by virtue of the strong intervalley $\left(\boldsymbol{K} \leftrightarrow \boldsymbol{K}^{\prime}\right)$ scattering at top adatoms, it is rapidly modulated on the atomic lattice as $U\left(\boldsymbol{r}_{i}\right) \sim-\cos \left[\left(\boldsymbol{K}-\boldsymbol{K}^{\prime}\right) \cdot \boldsymbol{r}_{i}\right]$, which yields a characteristic Kekulé pattern in the potential minima. This was recently shown to produce a robust "hidden" Kekulé ordering of certain types of impurities that survives even at room temperature [28].

Here we develop a derivation of the potential $U(\boldsymbol{r})$ using a simplified model for the adatoms. We describe graphene quasiparticles using a nearest-neighbor tight-binding model on the honeycomb lattice. The corresponding spin-degenerate Bloch Hamiltonian reads

$$
H_{0}(\boldsymbol{k})=-t\left(\begin{array}{cc}
0 & 1+e^{-i \boldsymbol{k} \cdot \boldsymbol{a}_{1}}+e^{-i \boldsymbol{k} \cdot \boldsymbol{a}_{2}} \\
1+e^{i \boldsymbol{k} \cdot \boldsymbol{a}_{1}}+e^{i \boldsymbol{k} \cdot \boldsymbol{a}_{2}} & 0
\end{array}\right),
$$

where $\boldsymbol{a}_{i}$ are the lattice vectors and the matrix is expressed in sublattice space, which we denote by $\alpha=$ A, B. The Hamiltonian of adatom $i=1,2$ is modeled as

$$
H_{i}=\epsilon_{0} .
$$

The hopping from graphene to adatom $i$ is expressed as a $1 \times 2$ hopping matrix from sublattice space to adatom level $\epsilon_{0}$. It may be either

$$
V_{i}^{A}=t(1 \quad 0)=t p_{A}
$$

for an adatom attached to the A sublattice or

$$
V_{i}^{B}=t\left(\begin{array}{ll}
0 & 1
\end{array}\right)=t p_{B}
$$

for the B sublattice.

The total energy of electrons scattering on two impurities at a distance $\boldsymbol{r}_{12}=\boldsymbol{r}_{2}-\boldsymbol{r}_{1}$ can be expressed as

$$
\begin{aligned}
U\left(\boldsymbol{r}_{12}\right) & =\int d \omega\left[\rho(\omega)-\rho_{\infty}(\omega)\right] \omega f(\omega), \\
\rho(\omega) & =-\frac{1}{\pi} \operatorname{Im} \operatorname{Tr}\left[G_{1}^{a}(\omega)+G_{2}^{a}(\omega)+\int d^{2} r G(\boldsymbol{r}, \boldsymbol{r} ; \omega)\right] .
\end{aligned}
$$

In this expression $f(\omega)$ is the Fermi distribution, and $\rho(\omega)$ is the total density of states at the energy $\omega$ of electrons in graphene (computed from the retarded Green's function $G$ of graphene) and in the two adatoms (computed from their respective $G_{1,2}^{a}$ ). The function $\rho_{\infty}(\omega)$ is the corresponding density of states for adatoms separated by a large distance (no interadatom scattering of electrons).

The graphene Green's function $G$ includes the coupling $V_{i}=V_{i}^{\alpha_{i}}$ of the two adatoms $i=1,2$ on sublattice $\alpha_{i}$ and can be derived using the Dyson equation. This yields

$$
\begin{gathered}
G\left(\boldsymbol{r}, \boldsymbol{r}^{\prime}\right)=g\left(\boldsymbol{r}, \boldsymbol{r}^{\prime}\right)+\sum_{i, j} g\left(\boldsymbol{r}, \boldsymbol{r}_{i}\right) T_{i j} g\left(\boldsymbol{r}_{j}, \boldsymbol{r}^{\prime}\right), \\
g\left(\boldsymbol{r}, \boldsymbol{r}^{\prime} ; \omega\right)=\sum_{s= \pm} e^{-i s \boldsymbol{K} \cdot\left(\boldsymbol{r}-\boldsymbol{r}^{\prime}\right)} \int \frac{d^{2} \boldsymbol{k}}{2 \pi} e^{-i \boldsymbol{k} \cdot\left(\boldsymbol{r}-\boldsymbol{r}^{\prime}\right)} g^{s}(\boldsymbol{k} ; \omega), \\
g^{s}(\boldsymbol{k} ; \omega)=\frac{1}{\omega-H_{0}(\boldsymbol{k})+i 0^{+}} .
\end{gathered}
$$

The $T$ matrix contains the scattering potential due to all possible inter- and intra-adatom scattering processes and reads

$$
\begin{aligned}
T_{i, j}(\omega) & =\left(\begin{array}{cc}
V_{1}^{\dagger} & 0 \\
0 & V_{2}^{\dagger}
\end{array}\right) \frac{1}{\left(\omega-\epsilon_{0}\right) \mathbb{1}-\Sigma^{a}(\omega)}\left(\begin{array}{cc}
V_{1} & 0 \\
0 & V_{2}
\end{array}\right), \\
\Sigma_{i j}^{a} & =\left(\begin{array}{cc}
V_{1} & 0 \\
0 & V_{2}
\end{array}\right)\left(\begin{array}{ll}
g_{11} & g_{12} \\
g_{21} & g_{22}
\end{array}\right)\left(\begin{array}{cc}
V_{1}^{\dagger} & 0 \\
0 & V_{2}^{\dagger}
\end{array}\right),
\end{aligned}
$$

where $g_{i j}=g\left(\boldsymbol{r}_{i}, \boldsymbol{r}_{j} ; \omega\right)$. The expression of $T^{\infty}$ and $G^{\infty}$ for adatoms infinitely apart is obtained simply by setting $g_{12}=$ $g_{21}=0$ above,

$$
\Sigma_{i j}^{a, \infty}=\left(\begin{array}{cc}
V_{1} & 0 \\
0 & V_{2}
\end{array}\right)\left(\begin{array}{cc}
g_{11} & 0 \\
0 & g_{22}
\end{array}\right)\left(\begin{array}{cc}
V_{1}^{\dagger} & 0 \\
0 & V_{2}^{\dagger}
\end{array}\right) .
$$

The adatom Green's function, on the other hand, reads

$$
G_{i}=\frac{1}{\omega-\epsilon_{0}-\Sigma_{i}^{g}},
$$

where the graphene-induced self-energy on adatom $i$ reads

$$
\begin{aligned}
\Sigma_{1}^{g} & =V_{1}^{\dagger}\left(g_{11}+g_{12} t_{2} g_{21}\right) V_{1}, \\
\Sigma_{2}^{g} & =V_{2}^{\dagger}\left(g_{22}+g_{21} t_{1} g_{12}\right) V_{2}, \\
t_{j} & =V_{j} \frac{1}{\omega-\epsilon_{0}-\Sigma_{j j}^{a, \infty}} V_{j}^{\dagger} .
\end{aligned}
$$

The asymptotic $G_{i}^{\infty}$ at large adatom separation is obtained by setting $g_{12}=g_{21}=0$ above.
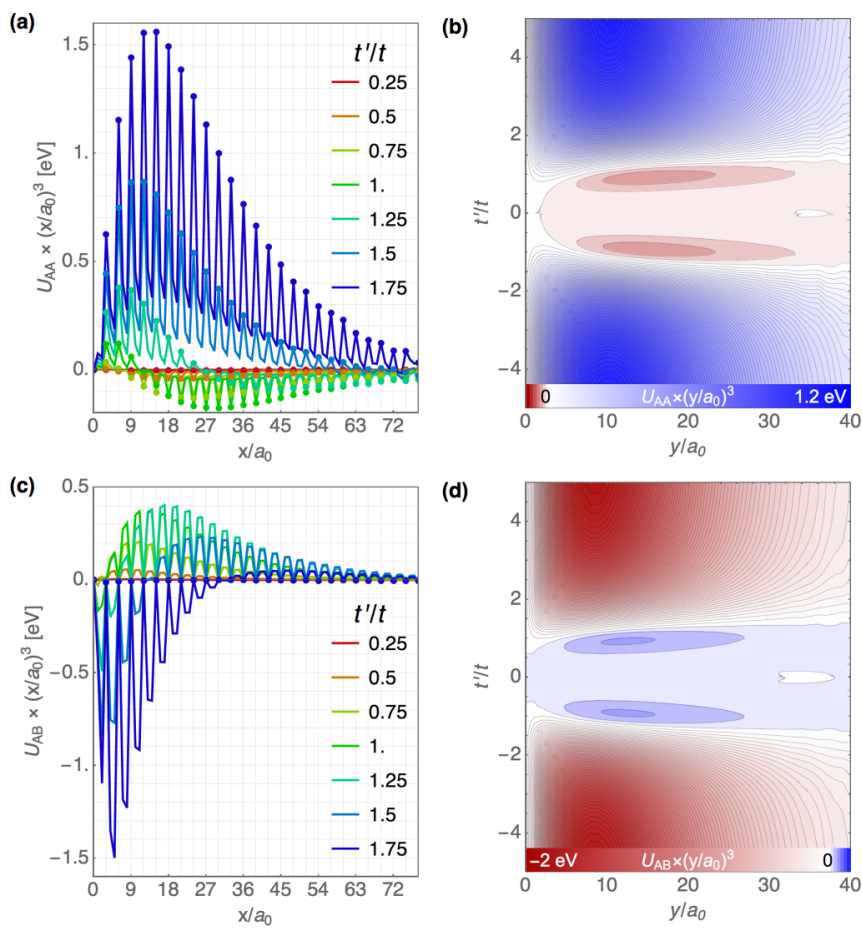

FIG. 4. Interaction potential (a) and (b) $U_{A A}$ and (c) and (d) $U_{A B}$ along the $x$ and $y$ directions at fixed $\epsilon_{0}=-0.15 t$ as $t^{\prime} / t$ is varied. The atomic-scale Kekulé oscillations arise only in cuts along the $x$ direction [(a) and (c)]. In (a) and (c), dots denote positions with $v_{r}=0$ (same Kekuké character of adatoms). $U_{A B}$ and $U_{A A}$ have opposite signs, but the sign is inverted in an attractive-repulsive crossover that appears around $\left|t^{\prime}\right| \approx 1.5|t|[31]$. 
(a)
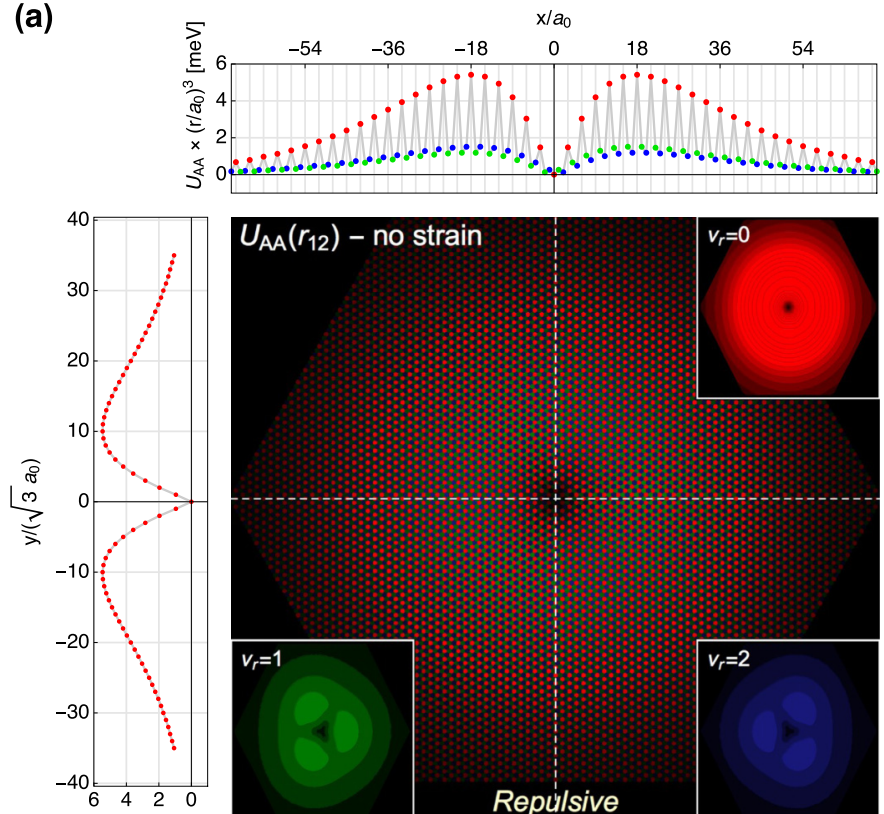

$U_{\mathrm{AA}} \times\left(\mathrm{r} / \mathrm{a}_{0}\right)^{3}[\mathrm{meV}]$

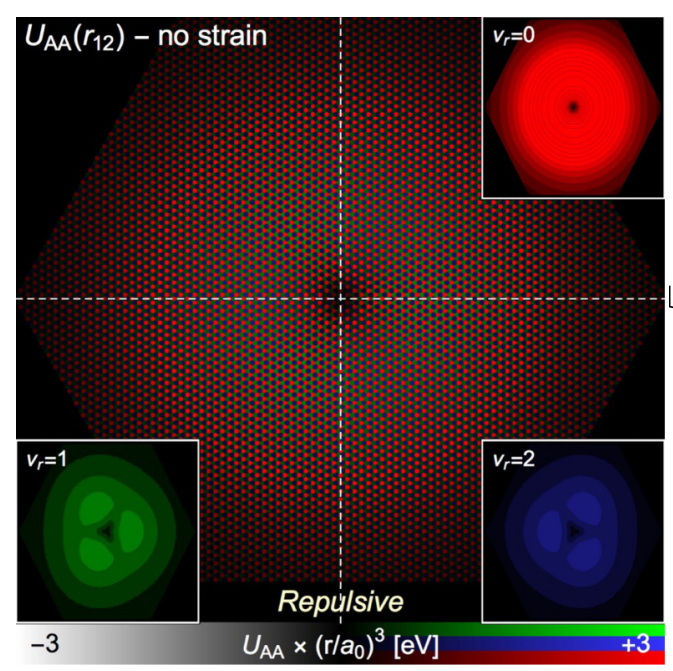

(b)
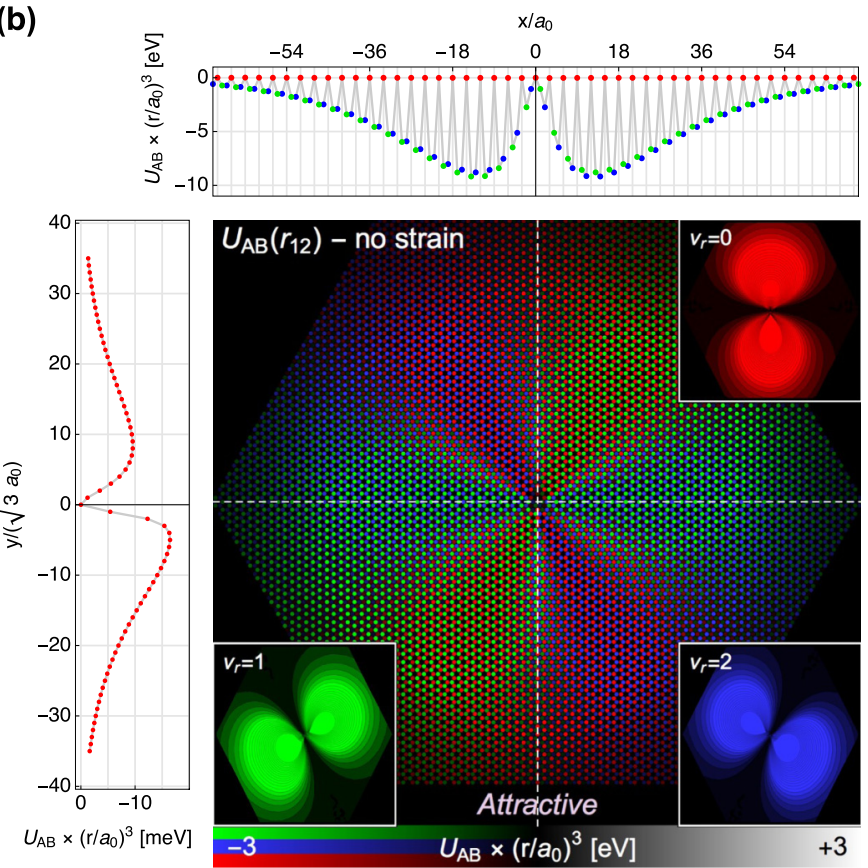

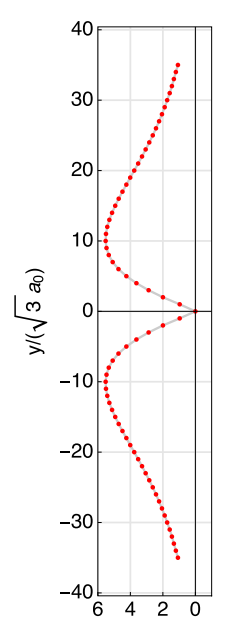

$U_{\mathrm{AA}} \times\left(\mathrm{r} / \mathrm{a}_{0}\right)^{3}[\mathrm{meV}]$
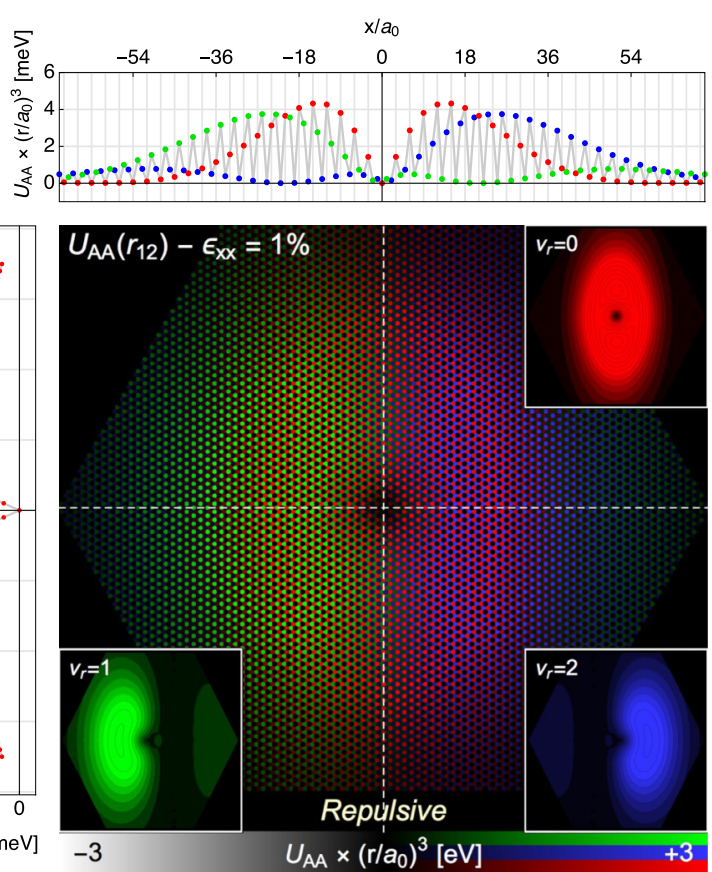

(d)
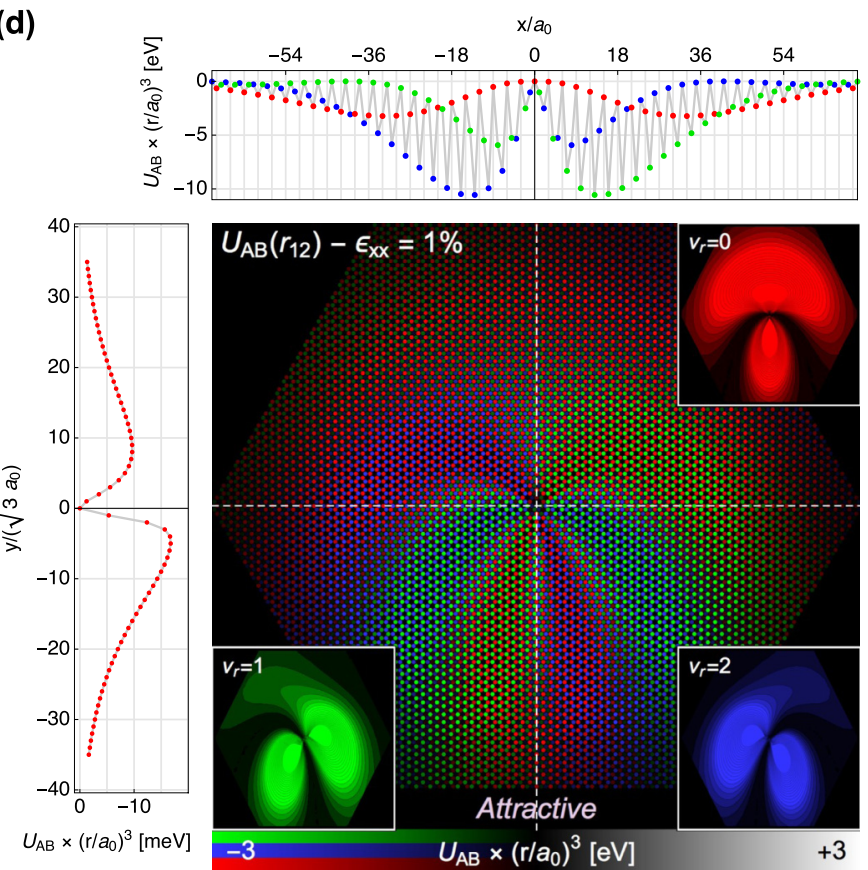

FIG. 5. Spatial map of the $U_{A A}(\boldsymbol{r})$ and $U_{A B}(\boldsymbol{r})$ adatom interaction in the strong-coupling limit $\left(t^{\prime}=5 t, \epsilon_{0}=-0.15 t\right)$. (a) and (b) The case without strain and (c) and (d) the case with a $1 \%$ uniaxial strain along the $x$ direction.

With these ingredients, the final form for the density of states reads

$$
\begin{gathered}
\rho(\omega)-\rho_{\infty}(\omega)=\Delta \rho^{g}(\omega)+\Delta \rho^{1}(\omega)+\Delta \rho^{2}(\omega), \\
\Delta \rho^{g}=-\frac{1}{\pi} \operatorname{Im} \operatorname{Tr} \sum_{s, i, j} \int \frac{d^{2} k}{2 \pi} g^{s}(\boldsymbol{k}) \Delta T_{i j} g^{s}(\boldsymbol{k}) e^{-i \boldsymbol{k} \cdot\left(\boldsymbol{r}_{j}-\boldsymbol{r}_{i}\right)}, \\
\Delta \rho^{i}=-\frac{1}{\pi} \operatorname{Im} \operatorname{Tr} \Delta G_{i},
\end{gathered}
$$

where $\Delta T_{i j}=T_{i j}-T_{i j}^{\infty}$ and $\Delta G_{i}=G_{i}-G_{i}^{\infty}$. Alternative derivations with different (but formally equiva- lent) forms of these equations can be found, e.g., in Refs. [29,31,38].

\section{APPENDIX B: STRONGLY COUPLED ADATOMS}

In the main text, we concentrated on the weak-coupling regime $t^{\prime} / t<1$ in which the Kekule interaction potential is attractive when the adatoms lie on the same sublattice and repulsive otherwise. This type of interaction has been observed experimentally at room temperatures for a specific type of "vacancy adatom" [28]. The magnitude and even the sign of the interaction, however, strongly depend on the ratio $t^{\prime} / t$, 
i.e., on how strongly the adatom or atomic defect binds to graphene. In this section we explore the dependence of the Kekule interaction as the coupling $t^{\prime}$ is increased. We find that the strong-coupling regime $t^{\prime} / t \gg 1$ is characterized by a repulsive Kekulé potential for same-sublattice configurations and an attractive potential for opposite sublattices, as noted by previous works [31]. The boundary between the weak-coupling and strong-coupling regimes is found at $t^{\prime} \approx 1.5 t$. This is shown in Fig. 4, where we plot the dependence of the $U_{A A}$ and $U_{A B}$ potentials along the $x$ and $y$ directions as one increases the ratio $t^{\prime} / t$ for a fixed $\epsilon_{0} \approx-0.15 t$.

Figure 5 shows the corresponding map for all interadatom distances $\boldsymbol{r}$ in the strong-coupling limit $t^{\prime}=5 t$. In Figs. 5(a) and 5(b) we show the case without any strain. We see that, indeed, same-sublattice interaction is now repulsive [Fig. 5(a)] and different-sublattice interaction is attractive [Fig. 5(b)]. Sublattice ordering will thus tend to be antiferromagnetic, with nearby adatoms arranging in opposite lattices. Without strain, different Kekulé alignments are favored [Fig. 5(b)], depending on the angle $\phi$ between $\boldsymbol{r}$ and $\Delta \boldsymbol{K}$ (here along the $x$ direction). The $U_{A B}$ potential that dominates the arrangement of adatoms is therefore nonisotropic, in contrast to the $U_{A A}$ potential that controls the weak-coupling regime. Most importantly, the magnitude of the adatom interaction is between one and two orders of magnitude stronger than in the weak-coupling regime.

In the presence of strain, the interaction potential becomes modulated following the same pseudogauge mechanism described in the main text. However, since adatom ordering in the strong-coupling regime is controlled by the nonistropic potential $U_{A B}$, the effect of strain has a much richer structure in this case [see Fig. 5(d)].

\section{APPENDIX C: INTERACTIONS}

Thus far, we have not considered the effects of electronelectron interactions in our discussion. In this section we consider intra-adatom Hubbard interactions in the weak-coupling limit. The Hubbard interaction introduces an additional term in the adatom Hamiltonian. In the mean-field approximation it is expressed as

$$
H_{j}=\epsilon_{0}+U \sum_{\sigma, \sigma^{\prime}}\left\langle n_{j, \sigma}\right\rangle n_{j, \sigma^{\prime}},
$$

where $U$ is the intensity of the Hubbard interaction, $n_{j \sigma}$ is the number operator for an electron in adatom $j=1,2$ with spin $\sigma=\uparrow, \downarrow$, and the magnetic moment in the adatom $M_{j}=$ $\left\langle n_{j, \uparrow}\right\rangle-\left\langle n_{j, \downarrow}\right\rangle$ is to be computed self-consistently.

Assume two adatoms $j=1,2$ on graphene. The mean value of the number of electrons with spin label $\sigma$ in adatom $j$ reads

$$
\left\langle n_{j \sigma}\right\rangle=-\frac{1}{\pi} \operatorname{Im} \int d \omega\left[\omega+i 0^{+}-H_{j}-\Sigma^{g r, j^{\prime}}(\omega)\right] \omega,
$$

and $\Sigma^{g r, j^{\prime}}(\omega)=-t^{\prime \dagger} G\left(\boldsymbol{r}_{j}, \boldsymbol{r}_{j}\right) t^{\prime}$ is the self-energy term accounting for the combined influence of the graphene lattice and the $j^{\prime} \neq j$ adatom on the $j$ adatom. The Green's function $G\left(\boldsymbol{r}_{j}, \boldsymbol{r}_{j}\right)$ of graphene under the $j$ adatom includes the presence of the $j^{\prime}$ adatom. It is computed by the same procedure explained in Appendix A for the noninteracting case, with the modification that the adatom Hamiltonian $H_{j}$ is now given by Eq. (C1) and is computed self-consistently. The formula for the potential $U$ given in the main text holds unmodified.
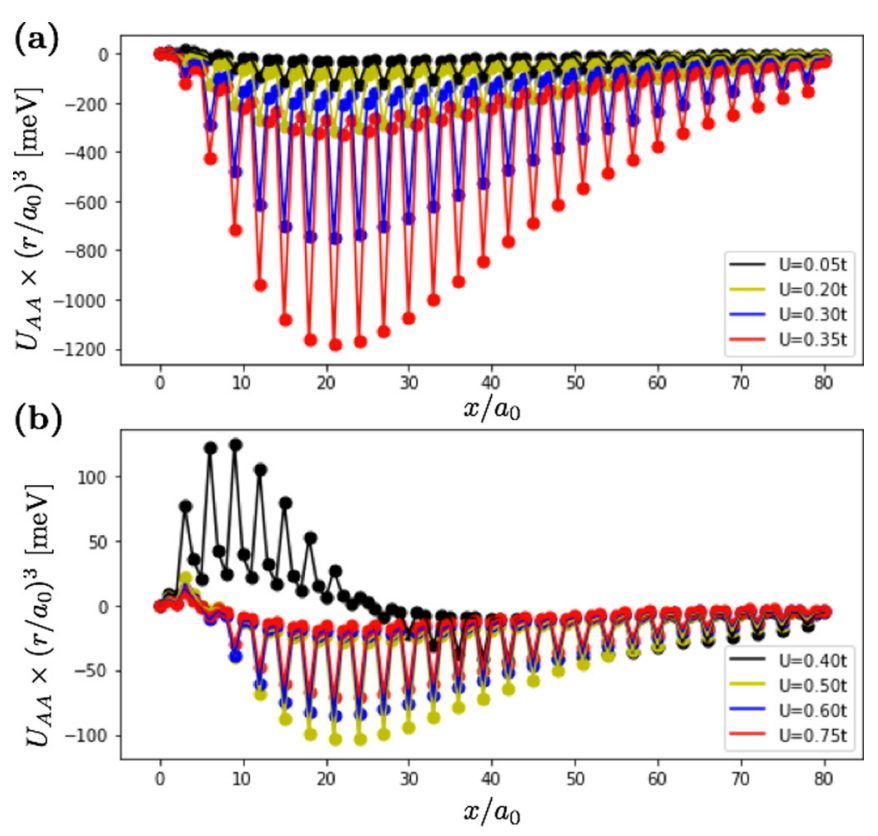

FIG. 6. Interaction potential for same-sublattice adatoms along the $x$ axis in the presence of an on-site electron-electron repulsion $U$. (a) The interaction potential for values of $U$ below $U_{c}$. (b) The evolution of the interaction potential in the ferromagnetic regime $U>U_{c}$.
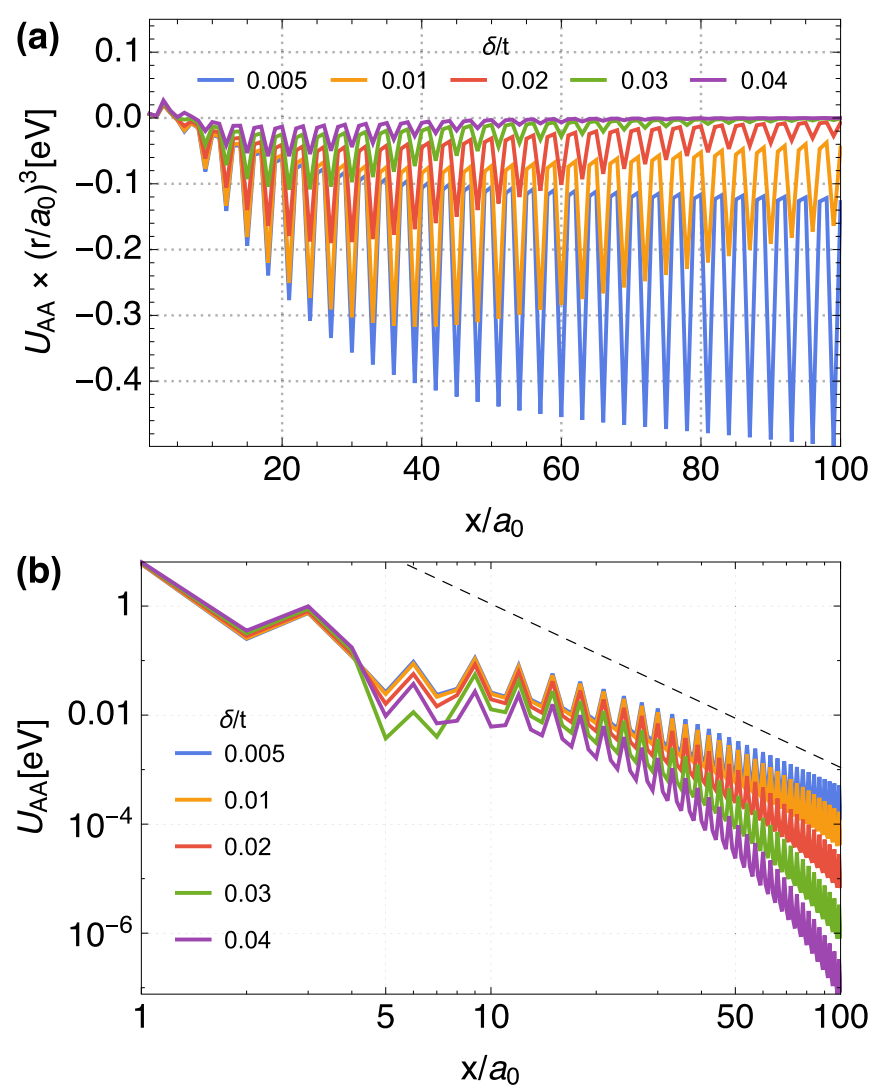

FIG. 7. $U_{A A}(r)$ cut at $y=0$ analogous to Fig. 2(a) in the main text as the damping factor of electrons in graphene is reduced from $0.04 t$ to $0.005 t$. The value chosen in the main text is $\delta=0.03 t$. Note that as coherence increases, the potential becomes stronger and decays as $1 / r^{3}$ (dashed line) at large distances. 
The spin-exchange interaction between magnetic adatoms in top positions is ferromagnetic when the adatoms are located in the same sublattice and antiferromagnetic when located in opposite sublattices $[17,44,45]$. We have confirmed this result within our model and have checked that the ferromagnetic character of the exchange remains unchanged under the application of strain in graphene. In the unstrained case, in the ferromagnetic regime the Kekule $\cos ^{2}(\Delta \boldsymbol{K} \cdot \boldsymbol{r})$ periodicity is left intact. Only the envelopes $v_{A A}$ and $v_{A B}$ of the oscillations are modified by the effect of $U$.

If the adatom is decoupled from graphene $\left(t^{\prime}=0\right)$, the presence of an arbitrarily small $U$ would open a spin-polarized splitting in the low-energy spectrum of the adatom. For $t^{\prime} \neq 0$, our mean-field approximation gives a minimum $U_{c}>0$ required to create a nonzero magnetic moment in the adatoms. For $t^{\prime}=0.7 t$ and $\epsilon=-0.15 t, U_{c} \approx 0.4 t$. Our numerical calculations show that the effect of the electron-electron repulsion is twofold. For $U<U_{c}$, the depth of the potential well increases with $U$, thus enhancing the attractive strength of the Kekule ordering. In the regime of ferromagnetic alignment $\left(U>U_{c}\right.$ ), the effect on the envelope is somewhat more complicated. For $U$ very close to $U_{c}$ the repulsive core around $\boldsymbol{r}=0$ is increased, although the interaction quickly becomes attractive for longer distances. Upon a further increase of $U$ the repulsive core shrinks dramatically, and the system returns to a behavior similar to the nonmagnetic case. This behavior can be observed in Fig. 6.

\section{APPENDIX D: DISSIPATION AND THE $1 / r^{3}$ ASYMPTOTICS}

It may be shown analytically [31] that a pristine and fully coherent graphene substrate leads to a same-sublattice adatom potential $U_{A A}(\boldsymbol{r})=v_{A A}(r) \cos \left(2 \pi v_{\mathbf{r}}\right)$ that scales asymptotically as $v_{A A}(r) \sim 1 / r^{3}$ with interadatom distance (at shorter distances, deviations are predicted depending on the adatom coupling strength [29]). This asymptotic result, however, assumes that dissipation is completely absent in the graphene electron liquid. Inelastic scattering events with phonons or through electron-electron interactions modify this result. In the main text, our simulations incorporated phenomenologically electronic dissipation by a finite imaginary part $\delta=0.03 t$ added to the energy $\omega+i \delta$ in the bare Green's functions $g$. The precise value of $\delta$ adequate for a real system is model dependent. Its effect on $v_{A A}$, however, is quite universal and leads to a suppression of the interaction strength and a faster decay than $1 / r^{3}$ at long distances. To make a connection to the analytical results for fully coherent systems we present in this section results for $U_{A A}(\boldsymbol{r})$ as the damping factor $\delta$ is reduced. Figure 7 shows cuts at $y=0$ analogous to those in Fig. 2(a) in the main text as $\delta$ is reduced from $0.04 t$ to $0.005 t$, both in a $U_{A A}(r) \times\left(r / a_{0}\right)^{3}$ plot [Fig. 7(a)] and in a log-log plot [Fig. 7(b)]. We see clearly that the interaction strength is enhanced as the system becomes more coherent and that the $1 / r^{3}$ decay [dashed line in Fig. 7(b)] is recovered.
[1] A. H. C. Neto, F. Guinea, N. M. R. Peres, K. S. Novoselov, and A. K. Geim, Rev. Mod. Phys. 81, 109 (2009).

[2] S. Das Sarma, S. Adam, E. H. Hwang, and E. Rossi, Rev. Mod. Phys. 83, 407 (2011).

[3] B. Amorim, A. Cortijo, F. de Juan, A. Grushin, F. Guinea, A. Gutiérrez-Rubio, H. Ochoa, V. Parente, R. Roldán, P. San-Jose, J. Schiefele, M. Sturla, and M. Vozmediano, Phys. Rep. 617, 1 (2016).

[4] K. Bolotin, K. Sikes, Z. Jiang, M. Klima, G. Fudenberg, J. Hone, P. Kim, and H. Stormer, Solid State Commun. 146, 351 (2008).

[5] C. Beenakker, Rev. Mod. Phys. 80, 1337 (2008).

[6] R. V. Gorbachev, J. C. W. Song, G. L. Yu, A. V. Kretinin, F. Withers, Y. Cao, A. Mishchenko, I. V. Grigorieva, K. S. Novoselov, L. S. Levitov, and A. K. Geim, Science 346, 448 (2014).

[7] I. Martin, Y. M. Blanter, and A. F. Morpurgo, Phys. Rev. Lett. 100, 036804 (2008).

[8] P. San-Jose and E. Prada, Phys. Rev. B 88, 121408 (2013).

[9] J.-H. Chen, W. G. Cullen, C. Jang, M. S. Fuhrer, and E. D. Williams, Phys. Rev. Lett. 102, 236805 (2009).

[10] A. Cresti and S. Roche, Phys. Rev. B 79, 233404 (2009).

[11] F. Libisch, S. Rotter, and J. Burgdörfer, New J. Phys. 14, 123006 (2012).

[12] J. A. Lawlor, S. R. Power, and M. S. Ferreira, Phys. Rev. B 88, 205416 (2013).

[13] L. Zhao, R. He, K. T. Rim, T. Schiros, K. S. Kim, H. Zhou, C. Gutiérrez, S. P. Chockalingam, C. J. Arguello, L. Pálová, D. Nordlund, M. S. Hybertsen, D. R. Reichman, T. F. Heinz, P. Kim,
A. Pinczuk, G. W. Flynn, and A. N. Pasupathy, Science 333, 999 (2011).

[14] I. A. Pašti, A. Jovanović, A. S. Dobrota, S. V. Mentus, B. Johansson, and N. V. Skorodumova, Phys. Chem. Chem. Phys. 20, 858 (2018).

[15] A. Pachoud, A. Ferreira, B. Özyilmaz, and A. H. Castro Neto, Phys. Rev. B 90, 035444 (2014).

[16] R. R. Nair, W. Ren, R. Jalil, I. Riaz, V. G. Kravets, L. Britnell, P. Blake, F. Schedin, A. S. Mayorov, S. Yuan et al., Small 6, 2877 (2010).

[17] H. González-Herrero, J. Gómez-Rodríguez, P. Mallet, M. Moaied, J. J. Palacios, C. Salgado, M. M. Ugeda, J.-Y. Veuillen, F. Yndurain, and I. Brihuega, Science 352, 437 (2016).

[18] A. F. Morpurgo and F. Guinea, Phys. Rev. Lett. 97, 196804 (2006).

[19] V. V. Cheianov, O. Syljuåsen, B. L. Altshuler, and V. Fal'ko, Phys. Rev. B 80, 233409 (2009).

[20] R. Balog, B. Jorgensen, L. Nilsson, M. Andersen, E. Rienks, M. Bianchi, M. Fanetti, E. Laegsgaard, A. Baraldi, S. Lizzit, Z. Sljivancanin, F. Besenbacher, B. Hammer, T. G. Pedersen, P. Hofmann, and L. Hornekaer, Nat. Mater. 9, 315 (2010).

[21] V. V. Cheianov, O. Syljuåsen, B. L. Altshuler, and V. I. Fal'ko, Europhys. Lett. 89, 56003 (2010).

[22] Y. Wang, S. Xiao, X. Cai, W. Bao, J. Reutt-Robey, and M. S. Fuhrer, Sci. Rep. 5, 15764 (2015).

[23] J. R. Wallbank, M. Mucha-Kruczyński, and V. I. Fal'ko, Phys. Rev. B 88, 155415 (2013).

[24] J. Jung, A. M. DaSilva, A. H. MacDonald, and S. Adam, Nat. Commun. 6, 6308 (2015). 
[25] S. Y. Zhou, G. H. Gweon, A. V. Fedorov, P. N. First, W. A. de Heer, D. H. Lee, F. Guinea, A. H. Castro Neto, and A. Lanzara, Nat. Mater. 6, 770 (2007).

[26] T. Iadecola, D. Campbell, C. Chamon, C.-Y. Hou, R. Jackiw, S.-Y. Pi, and S. V. Kusminskiy, Phys. Rev. Lett. 110, 176603 (2013).

[27] R. Lv, Q. Li, A. R. Botello-Méndez, T. Hayashi, B. Wang, A. Berkdemir, Q. Hao, A. L. Elías, R. Cruz-Silva, H. R. Gutiérrez, Y. A. Kim, H. Muramatsu, J. Zhu, M. Endo, H. Terrones, J.-C. Charlier, M. Pan, and M. Terrones, Sci. Rep. 2, 586 (2012).

[28] C. Gutierrez, C.-J. Kim, L. Brown, T. Schiros, D. Nordlund, E. B. Lochocki, K. M. Shen, J. Park, and A. N. Pasupathy, Nat. Phys. 12, 950 (2016).

[29] A. V. Shytov, D. A. Abanin, and L. S. Levitov, Phys. Rev. Lett. 103, 016806 (2009).

[30] V. Cheianov, V. Fal'ko, O. Syljuåsen, and B. Altshuler, Solid State Commun. 149, 1499 (2009).

[31] S. LeBohec, J. Talbot, and E. G. Mishchenko, Phys. Rev. B 89, 045433 (2014).

[32] V. V. Cheianov and V. I. Fal'ko, Phys. Rev. Lett. 97, 226801 (2006).
[33] A. Bácsi and A. Virosztek, Phys. Rev. B 82, 193405 (2010).

[34] J. Shi, B. Wu, X. C. Xie, E. W. Plummer, and Z. Zhang, Phys. Rev. Lett. 91, 076103 (2003).

[35] E. C. H. Sykes, P. Han, S. A. Kandel, K. F. Kelly, G. S. McCarty, and P. S. Weiss, Acc. Chem. Res. 36, 945 (2003).

[36] D. Zhabinskaya, J. M. Kinder, and E. J. Mele, Phys. Rev. A 78, 060103 (2008).

[37] D. Solenov, C. Junkermeier, T. L. Reinecke, and K. A. Velizhanin, Phys. Rev. Lett. 111, 115502 (2013).

[38] P. Hyldgaard and M. Persson, J. Phys.: Condens. Matter 12, L13 (2000).

[39] J. Bezanson, A. Edelman, S. Karpinski, and V. B. Shah, SIAM Rev. 59, 65 (2017).

[40] J. L. Mañes, Phys. Rev. B 76, 045430 (2007).

[41] I. Dzyaloshinsky, J. Phys. Chem. Solids 4, 241 (1958).

[42] T. Moriya, Phys. Rev. 120, 91 (1960).

[43] N. Nagaosa and Y. Tokura, Nat. Nanotechnol. 8, 899 (2013).

[44] A. M. Black-Schaffer, Phys. Rev. B 81, 205416 (2010).

[45] M. Sherafati and S. Satpathy, Phys. Rev. B 83, 165425 (2011).

[46] E. Kogan, Phys. Rev. B 84, 115119 (2011). 